ÉGYPTE monde arabe

\section{Égypte/Monde arabe}

12-13 | 1993

Une économie en transition

\title{
Répondre à la parole par la parole
}

Al-Cha'b, 16 juin 1992

\section{'Adil Husayn}

Traducteur : Muriel Berset

\section{(2) OpenEdition}

\section{Journals}

Édition électronique

URL : https://journals.openedition.org/ema/1288

DOI : 10.4000/ema.1288

ISSN : 2090-7273

\section{Éditeur}

CEDEJ - Centre d'études et de documentation économiques juridiques et sociales

\section{Édition imprimée}

Date de publication : 31 mars 1993

Pagination : 262-272

ISSN : 1110-5097

\section{Référence électronique}

'Adil Husayn, « Répondre à la parole par la parole », Égypte/Monde arabe [En ligne], 12-13| 1993, mis en ligne le 08 juillet 2008, consulté le 07 juillet 2022. URL : http://journals.openedition.org/ema/1288 ; DOI : https://doi.org/10.4000/ema.1288

Ce document a été généré automatiquement le 7 juillet 2022.

Tous droits réservés 


\title{
Répondre à la parole par la parole
}

\author{
Al-Cha'b, 16 juin 1992

\section{'Adil Husayn} \\ Traduction : Muriel Berset
}

1 C'est nous, et non pas vous, qui demandons de « répondre à la parole par la parole ». La sédition confessionnelle fait partie d'un complot, et seuls les imbéciles aident les comploteurs. Nous condamnons catégoriquement tout meurtre accompli sous le simple prétexte que la victime ne partageait pas notre foi ou nos opinions. C'est là un des principes fondamentaux de l'islam : « Soit croyant qui veut ; soit infidèle qui veut ». «Si ton Seigneur l'avait voulu, il aurait fait de l'humanité une seule communauté unie ».

2 En effet, l'islam tel que nous le concevons ne s'accomplira que si nous luttons pour la liberté de pensée. En outre, si ce principe est remis en question, nous sommes les premiers à en subir les conséquences. Nous combattons donc les démons, les orgueilleux et tous ceux qui veulent corrompre le monde.

3 Nous sommes vulnérables. Comment ne défendrions-nous pas le droit à la controverse, comment ne condamnerions-nous pas celui qui tue son contradicteur, alors que nous serions les premiers menacés si les armes faisaient la loi ? On ne peut donc pas mettre en doute notre condamnation de l'assassinat du Dr. Farag Fawda: nous sommes plus sincères que tous ceux qui poussent des hauts cris. La preuve de notre bonne foi réside dans notre position ferme pour ce qui concerne les droits de l'homme, tandis que les amis de F. Fawda, eux, font deux poids deux mesures.

4 «Répondre à la parole par la parole », et non par la violence ou les effusions de sang: tel est le principe qui ressort de tout ce qui a été publié par les amis de Farag Fawda après ce crime déplorable. Nous sommes parfaitement d'accord avec ce principe, nous l'avons déjà mentionné plus haut. Mais la liberté d'opinion est-elle absolue ? N'est-elle pas, dans tous les pays du monde, limitée par des lois ou des coutumes? En effet, en tout lieu et à toute époque, le penseur tient compte du contexte et respecte les sentiments et croyances dominants. En Égypte, il s'agit en premier lieu de l'islam. Si les écrits qui traitent de l'islam ne sont pas inspirés par la foi, qu'au moins ils en parlent avec respect et en connaissance de cause, de sorte qu'on puisse dire ensuite: ils ont répondu à un argument par un argument. Bien que les écrits du Dr. Fawda (Que Dieu lui 
pardonne !) n'aient pas répondu à ces impératifs - la rigueur scientifique et le respect dans le dialogue - nous n'avons jamais réclamé que les médias officiels cessent de le publier, et nous ne lui avons jamais souhaité cette fin tragique.

5 Nous adressons maintenant cette question aux amis du disparu: lorsque vous approuvez cette manière - non respectueuse - de traiter de l'islam, vous faites-vous les défenseurs d'une liberté d'expression totale, qui n'est soumise à aucune norme scientifique ni à aucun respect des convenances? Si c'est le cas, pourquoi ne revendiquez-vous pas, comme nous, qu'on nous accorde davantage de liberté dans tous les domaines ? Pourquoi passez-vous sous silence les atteintes à la liberté lorsqu'elles sont le fait des autorités et des corrupteurs ? Pourquoi ne réclamez-vous que la liberté de dénigrer l'islam et ses figures emblématiques? L'islam est-il devenu le bouc émissaire? Quand vous parlez de la nécessité de "répondre à la parole par la parole ", pourquoi ne réclamez-vous pas que nous soit octroyé, à nous aussi, le droit dont vous jouissez vous-même de vous exprimer à la télévision et dans les grands quotidiens ? Et puisque nous sommes bannis de ces institutions, qui donc, dans cette situation, défend le principe «Répondre à la parole par la parole »? Le gouvernement et vous, ou bien nous? Si j'ai la possibilité d'écrire dans mon propre journal (et que Dieu me conserve ses faveurs), qu'en est-il pour ceux qui n'ont même pas cette chance?

6 A-t-on donné aux partisans des idées extrémistes l'occasion de les exposer, de les expliquer dans la légalité ? Ils ont donc continué à agir clandestinement et à ouvrir le feu. Leur avez-vous demandé de s'expliquer, vous qui êtes épris de liberté, afin d'isoler et d'anéantir les germes de violence et de sédition? Ou bien la liberté n'est-elle pour vous que le privilège d'un seul bord ? Vous parlez de liberté de pensée ? Très bien, nous approuvons. Mais alors, ne voyez-vous pas que toute une frange des intellectuels islamistes de cette nation est condamnée au silence ? Vous l'insultez, vous la calomniez sans discontinuer tandis qu'elle ne peut ni répondre ni s'expliquer!

7 Nous nous joignons cependant à vous pour demandera ces jeunes de faire preuve de patience et de ne recourir ni à la violence ni aux effusions de sang. Mais la différence entre vous et nous est que pour notre part, nous mesurons tout à la même aune : nous réclamons donc la même chose du gouvernement et de ces jeunes. Si nous nous opposons à la violence de ces derniers, nous exigeons également du gouvernement qu'il interdise la torture et l'usage meurtrier des armes à feu, pratiques totalement illégales. Nous avons déjà démontré, faits et chiffres à l'appui, que ce qui se produit actuellement dépasse en étendue et en horreur tout ce que nous avons pu vivre ou entendre raconter au cours de notre histoire. Pour être plus précis, les tortures et mauvais traitements pratiqués actuellement dépassent ceux des années 60 , qui n'en avaient pourtant pas été avares. "Épris de liberté ", cette remarque vous émeut-elle? Avez-vous réclamé la création d'un comité national d'enquête qui fasse la lumière sur ces pratiques criminelles, vous qui clamez - mensongèrement - que vous voulez qu'on réponde à la parole par la parole? Pourquoi deux poids deux mesures? Pourquoi accepter tel meurtre et condamner tel autre?

8 J'ai une autre remarque à faire, adressée à Al-Ahâlî cette fois : on a l'impression, dans le flot des adieux et condamnations [ayant suivi l'assassinat de Farag Fawda]qu'il n'existait aucune divergence d'opinion entre cet hebdomadaire et l'écrivain assassiné. Or, nous continuons à penser que ces divergences étaient nombreuses.

9 Le concept (marxiste) de " contradiction principale », qui était encore en vigueur il n'y a pas si longtemps, signifie, dans le cas de l'Égypte, que le front du conflit en cours (la 
contradiction principale) est la lutte contre les Etats-Unis et Israël, le reste étant considéré comme une "contradiction secondaire" au sein des masses. Malgré la disparition de nombreux concepts marxistes, cette conception des choses n'a pas changé et demeure utile pour l'expérience du mouvement nationaliste. Certes, si les communistes considéraient les Etats-Unis et Israël comme des ennemis, ce n'est pas en relation avec l'URSS disparue, mais en relation avec leur « nation » immuable qui, elle, existe toujours. Si tout cela est vrai, les auteurs à Al-Ahâlînt-ils oublié quelle était la position de Farag Fawda en ce qui concerne l'Égypte et les Arabes? Ont-ils oublié qu'il était le partisan de l'hégémonie américano-sioniste et de ses plans? Certes, Farag Fawda n'a pas besoin d'être le champion du nationalisme pour que nous condamnions son assassinat, mais quelles que soient votre émotion et votre tristesse, elles ne justifient pas que vous oubliez tout critère de jugement.

\section{La sédition confessionnelle, ou l'unité nationale... sans les musulmans !}

10 Venons-en maintenant au problème de la sédition confessionnelle. Les alarmistes n'ont pas manqué d'en parler ces dernières semaines et ils ont déjà injecté leur venin. Ils sont parvenus à des conclusions inquiétantes. De même que, lors de l'affaire Farag Fawda, ils se sont proclamés à tort défenseurs de la liberté d'opinion et partisans du principe «la parole en réponse à la parole », de même ils se présentent, quand ils évoquent la sédition confessionnelle, comme les champions de l'unité nationale... En réalité, c'est nous qui la défendons. Ils déclarent suivre l'opinion de leur défunt chef de file, Farag Fawda. Or, quelle conception de l'unité nationale avait M. Fawda? Il la résumait en ces mots : «alliance des laïcs et des chrétiens »... Voilà le moyen le plus sûr d'aboutir à la sédition et l'assurance de ne pas réaliser l'unité en question: M. Fawda écarte purement et simplement les musulmans : quelle "unité " est-ce là ? La sédition ne consiste-t-elle pas, justement, à plonger les chrétiens dans un conflit avec la majorité de la nation? Et quel meilleur moyen existe-t-il, pour faire s'enliser les frères chrétiens dans cette alliance, que de leur faire craindre les musulmans et d'exciter les passions? C'est d'ailleurs bien ce à quoi œuvrent les partisans de la laïcité.

Comment envisageons-nous, pour notre part, la question de l'unité nationale? Je me suis déjà maintes fois exprimé à ce sujet, mais puisque la situation s'aggrave, je vais me répéter et préciser ma pensée... Nous devons actuellement affronter un danger considérable : celui que représentent - en premier lieu - les puissances étrangères qui ne veulent aucun bien à l'Égypte ni aux Arabes. Ces puissances ont attaqué l'Irak, occupé le Golfe, menacent maintenant la Libye. Elles ont anéanti l'économie égyptienne, l'ont livrée aux sionistes américains. Elles travaillent à diviser les sociétés arabes en factions ennemies : en Irak, elles soutiennent les Kurdes contre les Arabes, montent les sunnites contre les chiites et réciproquement. Au Liban, nous assistons depuis 17 ans à une sanglante tragédie. Au Soudan sévit depuis dix ans une guerre civile entre le Nord et le Sud. En Égypte existe un plan visant à diviser musulmans et chrétiens, et rien n'empêche que les Nubiens n'entrent dans le jeu à un moment ou à un autre. 


\section{Les comploteurs et les imbéciles}

12 Ce complot ne peut qu'être appuyé par des groupes internes, chrétiens ou musulmans. Il est de notre devoir - pour tuer dans l'œuf la sédition - d'examiner ce complot pour en comprendre les tenants et les aboutissants et lui opposer une ligne de conduite nationale. Les médias officiels ne cessent d'évoquer la «main de l'étranger » qui finance et manipule les éléments musulmans afin de provoquer la sédition. Ceux qui tiennent ce genre de propos entendent en général par « étrangers » l'Iran et le Soudan, ce qui, franchement, prête à rire : ainsi certains auteurs demandent-ils que l'armée égyptienne cesse d'être mobilisée contre Israël afin d'être disponible pour une guerre contre le Soudan! Or, en matière de sédition confessionnelle, justement, c'est au complot sioniste, et non à ce qui se passe au Soudan, que nous devons accorder toute notre attention. Quant à ceux qui donnent ces conseils douteux, ils organisent des rencontres avec les sionistes et les Américains en vue de coordonner leurs actions.

Il nous appartient d'expliquer ce qui se passe côté musulman: nous exigeons que davantage de recherches soient effectuées dans cette direction, et ceci sans honte. Cependant, nos frères chrétiens font preuve d'une pudeur extrême lorsque nous leur demandons d'analyser ce qui se passe de leur côté. Or l'ennemi concentre ses visées surtout sur eux, puisqu'il se considère comme "le protecteur des minorités ", ce qu'il n'a cessé de prétendre depuis l'époque de la colonisation anglaise et qu'il a d'ailleurs formulé explicitement dans la célèbre déclaration du 28 février (les coptes s'y sont à ce moment-là opposés avec courage et fermeté). Les chrétiens refusent maintenant de se soumettre à la rigueur scientifique, et lorsque le $\mathrm{D}$. Rafiq Habib (un véritable chercheur, chrétien et nationaliste) a tenté de le faire, il a été victime d'une étrange persécution issue de l'intérieur et menacé d'excommunication. Il a fait part de son expérience dans son dernier livre : Le meurtre d'une génération - l'Église et le retour de l'inquisition.

Nous désapprouvons cette manière de vouloir dissimuler la vérité : à notre avis, une telle confusion ne peut que nuire au respect mutuel que se doivent les fils de la nation et faire obstacle à la réalisation d'une unité nationale organique.

Il y a déjà des années de cela, le Dr. Milad Hanna nous a informés, dans son livre : Coptes, oui... mais Égyptiens,qu'existe un courant rassemblant des coptes convaincus que l'intérêt de l'Égypte réside dans un engagement aux côtés de l'Occident, ce dernier défendant ce que Churchill appelle " notre civilisation chrétienne ». Ils considèrent par conséquent que s'éloigner du monde arabe va affaiblir l'islam, et ils sont attachés à la paix avec Israël.

\section{L'évangélisation et le pape américain}

16 La tendance [dont parle Milad Hanna] devient encore plus dangereuse lorsqu'elle cesse d'être un courant purement politique pour devenir croyance religieuse. Et contrairement à une conviction fort répandue, l'activité missionnaire n'est pas tant axée sur les musulmans que sur les coptes: il s'agit de leur faire abandonner leurs convictions traditionnelles, hostiles à Israël et au christianisme' occidental. Le Dr. Rafiq Habib nous a clairement démontré, dans son ouvrage (Le christianisme et la guerre: histoire du fondamentalisme sioniste américain et du combat contre l'Orient musulman),que les organisations fondamentalistes américaines parvenaient effectivement à pénétrer nos 
différentes églises (orthodoxe, protestante, catholique) par un travail de l'intérieur et par l'intermédiaire de camps basés à l'étranger. Elles sont ainsi parvenues à « rééduquer » religieusement et socialement un grand nombre de jeunes. N'avons-nous pas entendu parler du christianisme sioniste (une branche du fondamentalisme américain) qui croit à la nécessité de détruire la mosquée Al-Aqsa, de reconstruire le Temple de Salomon et de faire émigrer les juifs en Israël afin de préparer le retour du Messie, qui devra régner sur la terre pendant 1000 ans ? Cette secte, que les chrétiens égyptiens (toutes églises confondues) ne connaissaient pas, a maintenant des adeptes qui s'efforcent de l'implanter et d'en diffuser les principes. Ikram Lam'i, prêtre et auteur d'un ouvrage intitulé $Y$ a-t-il un lien entre le retour des juifs et la seconde venue du Messie ?, y déclare que

«cette croyance influence la conscience de ses fidèles et leur pose un dilemme spirituel et moral : un pilote chrétien adepte de cette secte m'a expliqué que si on lui donnait l'ordre d'attaquer Israël, il l'exécuterait et combattrait pour son pays, car il est patriote, mais en même temps, il est convaincu qu'Israël finira immanquablement par gagner. Comment peut-il être à la fois fidèle à son pays, à ses sentiments patriotiques et à sa foi. "

17 "Ce phénomène,écrit Rafiq Habib, gagne en importance en raison de la progression du millénarisme, dont les adeptes augmentent, y compris au sein des confessions qui rejettent cette croyance. » Les tentatives effectuées pour influencer les coptes d'Égypte ne se limitent pas à cette activité missionnaire, qui dispose de moyens énormes; il faut y ajouter l'installation à l'étranger des communautés d'émigrants. Certaines sont devenues complices d'entreprises évidentes de sabotage, telles celles qui projettent de créer un état copte indépendant sur le territoire égyptien!

Puisque nous parlons des colonies de coptes émigrés, je rapporte ici l'avertissement lancé par le professeur Galam Kichk. En effet, ce dernier tient beaucoup à l'ancrage national de l'Église-Mère - l'Église orthodoxe de Marc - mais il déclare :

«Les mots «Église copte» ne coïncident plus avec l'Église égyptienne, devenue plurinationale. Il est à craindre que ceux de nos compatriotes nés à l'étranger ou émigrés à un âge précoce perdent un jour, peut-être, leur identité égyptienne et orientale. Alors des Égyptiens qui ne le sont plus ni par appartenance ni par loyauté deviendront prêtres, évêques, archevêques... À l'étape suivante, l'Église de St. Marc aura à sa tête un pape américain ou australien. Alors sera réalisé ce à quoi ont échoué les Tsars. N'oublions pas que l'Église américaine a réussi à s'imposer jusque dans le cénacle du Vatican et influence le choix des papes. "

19 (Lire le livre: Ne sont-ils pas tombés dans la sédition?) Nous devons donc prêter attention à ce qui se passe autour de nous si nous voulons vraiment arrêter la sédition à sa source et faire échouer les complots. L'ennemi - les faits et la logique le prouvent - a pénétré les rangs des musulmans comme des chrétiens. Il doit inévitablement exister des réseaux organisés et très actifs qui suivent des directives visant à provoquer une sédition. II est manifeste que ces groupes seront de plus en plus actifs si personne ne s'interpose. Cependant, leur influence reste encore limitée car les imbéciles ne sont jusqu'à maintenant qu'une poignée parmi les croyants de toutes confessions. Par «imbéciles»- ne soyez pas choqués - nous entendons ici ceux qui réagissent favorablement aux instigations des agents de ces réseaux, et qui servent les intérêts de l'ennemi du fait de leur ignorance et de leur naïveté. N'épargnons pas les musulmans « déviants »: il n'est plus temps d'échanger des congratulations. Al-Cha'ba adressé des critiques directes aux musulmans et chrétiens mêlés aux escarmouches qui ont éclaté entre les deux communautés. Certains groupes islamistes se sont comportés avec 
stupidité - et non pas "folie", comme le laissent entendre les médias officiels : l'homme stupide réagit à un tort réellement éprouvé, mais il réagit impétueusement, sans preuve et de façon disproportionnée. Le dément frappe sans prévenir et sans raison. Mais les médias officiels et les auteurs anti-islamistes présentent habituellement ces incidents comme des actes de pure folie dus au «fanatisme ». Ces incidents étant récurrents, ils semblent faire naturellement partie de l'islam, en être indissociables. Alors que des membres de la sécurité et des instances dirigeantes affirment en toute bonne foi que les événements récemment survenus à Dayrout sont pour une bonne part liés à la vendetta, c'est-à-dire à une pratique sociale typique du Sa'id, nos amis refusent de croire qu'il s'agit simplement d'un comportement traditionnel absurde et insistent pour y voir la preuve d'un fanatisme qui n'existerait que parmi les musulmans. Al-Cha'ba pourtant indiqué, dans ses reportages sur les incidents récents, que des éléments provocateurs y étaient mêlés.

Certains jeunes islamistes se sont comportés de manière brutale et ont effrayé des chrétiens paisibles, menaçant leur vie ou leurs biens, ce qui est absolument contraire aux principes de l'islam et aux traditions de respect mutuel et de fraternité. Leur stupidité réside dans le fait que, d'une part, leur réaction était sans proportion avec l'incident qui l'avait suscitée et que, d'autre part, la provocation peut venir d'un agent (peut-être musulman) dont l'objectif est d'inciter à la violence. Ainsi, les extrémistes présents dans nos rangs réalisent ses desseins et nuisent - alors qu'ils s'imaginent avoir agi pour le mieux - à la réputation des musulmans et au pouvoir islamique auquel nous aspirons.

21 Nous entendons parler de propagande missionnaire visant à convertir les musulmans au christianisme; des publications allant dans ce sens seraient en circulation; des fonds seraient récoltés auprès des coptes pour préparer une guerre contre les musulmans : nous en avons rendu compte. Al-Ahrâm,en avril 91, a annoncé l'arrestation de ressortissants suisses et allemands qui, ayant loué un appartement à 'Agouza, avaient noué des contacts dans la population en vue de faire douter les musulmans de l'islam et de les convertir au christianisme. Voilà une nouvelle révoltante! Mais que peuvent les coptes égyptiens à tout cela? Il faut à tout prix considérer avec fermeté ces manoeuvres douteuses, il faut estimer les dangers à leur juste mesure afin que la sédition ne s'aggrave pas (ce qui servirait les objectifs de l'ennemi) alors que nous voulons lutter pour l'éliminer.

J'affirme donc que le fanatisme et la stupidité existent aussi bien du côté chrétien que du côté musulman. Grâce à Dieu, une autre personne partage mon opinion, sa Sainteté le pape Chenouda lui-même, qui a dit : «Le fanatisme peut apparaître en tout lieu, chez tout peuple et toutes tendances. "Cependant, Sa Sainteté insiste sur le fait que le "fanatisme mêlé de violence " n'existe pas chez les coptes mais seulement chez les islamistes, qui sont allés jusqu'à assassiner un ouléma musulman (le cheikh alDhahabi). Et Sa Sainteté s'en tient à cette position bien que son porte-parole lui ait rappelé que l'Association de la Nation Copte avait, en 1954, enlevé sous la menace des armes al-Anba Kasab et l'avait contraint à renoncer à son statut papal (voir le livre de Mahmud Fawzi : Le pape Chenouda et l'opposition au sein de l'Église). 


\section{Nous voulons un dialogue sensé entre patriotes des deux bords} mais, en premier lieu, aux musulmans et chrétiens les plus sensés et les plus loyaux: s'ils parviennent à trouver un terrain d'entente, il nous sera alors possible de corriger les déviations et de contenir les impulsifs et les fanatiques des deux bords. Je suppose qu'il existe, côté musulman, des figures emblématiques de la raison et de la souplesse dont les propos sont clairs, et j'espère que nous trouverons un interlocuteur chrétien prêt à serrer la main tendue.

désirons un dialogue juste et fructueux, il faut d'abord en finir avec l'idée d'une alliance entre laïcs et chrétiens, idée qui n'a aucun fondement et anéantit l'objectif d'unité nationale que réclament - sans doute aucun - la majorité des coptes. En effet, la laïcité écarte de la vie sociale et politique la religion et ses valeurs ; en d'autres termes, elle marginalise la religion. Donc, si l'alliance en question était réalisée, il s'ensuivrait une marginalisation de l'islam lui-même, et non du christianisme qui serait, lui, représenté par ses institutions et ses autorités religieuses et politiques. Il s'agit là d'une provocation non dissimulée à l'égard des musulmans. Si encore il était question d'écarter les autorités religieuses des deux bords, il y aurait au moins une logique (bien que nous rejetions cette option). Mais on ne peut écarter les oulémas et les intellectuels musulmans et garder le pape Chenouda tout seul au nom de l'unité nationale! Ce n'est ni sensé ni acceptable! Et comme nous l'avons dit plus haut, quel sens peut avoir une « unité » qui ignore $90 \%$ de la population (et nous ne disons pas $94 \%$ !). Cette idée d'alliance entre laïcs et chrétiens réapparaît régulièrement dans les demandes de suppression des programmes islamiques télévisés (peu nombreux) et des rubriques islamiques de la presse quotidienne, etc., cela sous prétexte que les responsables de ces programmes et rubriques sont des " fanatiques »: ceux qui s'y intéressent ont pourtant remarqué que ces responsables sont soumis à un contrôle qui leur impose un ton résolument modéré. Et pour reprendre les paroles de Sa Sainteté le pape, si ce qui lui importe est l'incitation à la violence et non l'extrémisme d'opinion, nous pouvons constater que les médias officiels ne laissent passer aucun appel à la violence et, au contraire, ne cessent de la condamner. Certes, ce qui nous est proposé à la télévision et dans la presse officielle est loin d'être parfait; or nos amis ne demandent pas d'en corriger les insuffisances, mais bien de supprimer tout ce qui contribue à propager la pensée religieuse - ou ce qui touche au Mystère (ghayb)comme ils disent.

L'alliance laïcs-chrétiens n'écarte pas seulement les islamistes fanatiques, apôtres de la violence, mais également les figures emblématiques de la raison, qui connaissent bien la pensée islamique (les cheikhs al-Ghazali, al-Cha'rawi, Gadd al-Haqq, Fahmi Huwaydi, Muhammad 'Imara, Abu-l-Magd, etc., sans compter le Parti du Travail et les Frères musulmans). Nous déclarons aux patriotes coptes sensés que les laïcs ne représentent qu'une frange très étroite d'intellectuels de salon et qu'une alliance avec eux ne garantirait ni stabilité ni sécurité. Tant que vous ne prendrez pas en considération les musulmans dans leur ensemble, tant que l'entente mutuelle n'est pas réalisée sur une base claire et juste, la voie sera libre pour les conspirateurs et les imbéciles. Une telle entente n'est pas possible si les coptes éclairés posent comme condition à l'unité nationale le rejet de l'islam et l'abandon de la charîa.Celui qui tient de tels propos annihile du même coup toute chance d'entente. Jamais les dizaines de millions de

Égypte/Monde arabe, 12-13 | 1993 
citoyens musulmans ne l'accepteront. Nous devons donc cohabiter et coopérer en tenant compte de cette réalité (comme cela a été le cas au cours de notre histoire).

J'ajouterai que les discours que tient sa Sainteté le pape Chenouda sur la signification de la charî'a,et son application ne sont pas, en tout cas, très heureux (voir Le pape Chenouda et l'opposition...).Je n'ajouterai rien de plus. Néanmoins, je dirai que les propos qu'il a tenus lors d'un entretien publié dans Al-Ahâl(t(janvier 90) sont très décevants. Nous n'affirmerons jamais assez combien nous approuvons, en revanche, ceux de Makram 'Ubayd (le grand leader nationaliste) : «Je suis musulman de patrie, chrétien de religion. " Il voulait dire par là que le patrimoine musulman est la source commune à laquelle s'abreuvent les fils de la Nation, chrétiens et musulmans. Le pape nous a déçus en manifestant son désaccord avec Makram 'Ubayd: il a ainsi ébranlé les fondements historiques, culturels et affectifs de notre unité nationale, et nos liens arabes avant la révolution de 1919 et après.

Ces prises de position papales prennent une signification profonde lorsqu'on observe le comportement effectif de l'Église ou ce qui en apparaît: elle applique en effet l'idée d'une alliance laïcs-chrétiens, de même qu'elle soutient les laïcs qui attaquent l'islam et provoquent les musulmans, alors qu'elle interdit le dialogue et les rencontres avec toutes les grandes figures islamiques. Nous demandons donc aux nationalistes coptes sensés (ils sont la majorité) de revenir sur cette position. Qu'ils voient la main loyale qui leur est tendue. Il est de votre droit incontestable d'attendre de nous de solides assurances, et je jure par Dieu que si nous avons des principes islamiques (au sens où nous les comprenons) vous trouverez sécurité et justice. Nous espérons que vous nous écouterez afin que disparaissent les hantises et que se taisent les appréhensions. Nous vous invitons à participer à la vie sociale et politique, et le Parti du Travail vous ouvre ses portes.

Croyez-moi, parmi nous, les fanatiques pathologiquement atteints sont une minorité, et nous devons les affronter ensemble, comme des citoyens également désireux d'édifier la patrie. Les musulmans ont davantage de devoirs vis-à-vis de ce problème? Assurément, mais n'exagérez pas, pour votre part, votre peur et votre isolement parce que vous vous sentez minoritaires. En effet, la non participation des coptes à l'effort général inspire une profonde inquiétude à notre parti et aux forces nationalistes sensées. Nous comprendrions que vous vous teniez éloignés seulement du Parti du Travail, étant donné son orientation islamiste; or vous vous retirez de toute participation politique, même au sein du parti gouvernemental, car vous avez mis le président Moubarak dans le même sac que nous (c'est-à-dire les « extrémistes »). C'est bien la première fois que nous nous trouvons dans le même sac! Ce phénomène de retrait devient manifeste et inquiétant; il s'est transformé en une main-mise de l'Église sur l'activité politique, qui a maintenant pris le pas sur tout autre. Espérons qu'elle revienne à son rôle naturel, celle d'institution religieuse nationale, et laisse la politique et les partis au libre choix de ses ouailles.

29 Ai-je dépassé les bornes ? Je ne le crois pas. Mais si c'est le cas, la situation actuelle est tellement inquiétante qu'il nous incombe de nous exprimer librement et sincèrement afin de lutter contre la sédition. En ce qui concerne, en particulier, le rôle politique de l'Église, je voudrais souligner ce que mon frère Philippe Galâb a écrit à ce sujet. J'ai pu lire des propos de même nature dans le dernier numéro d'Al-Ahâlîsous la plume de mon ami Gamal As'ad. Quant aux amis Milad Hanna et Magid 'Attiyya, je me réjouis d'avoir vu dernièrement leur signature dans le journal Watani: cela signifie un rôle politique 
accru pour les chrétiens (hors du clergé). Je pense avoir raison, mais si ce rôle doit être à la mesure de ce que j'ai lu dans les deux derniers numéros, alors on peut s'en passer ! Milad a révélé un côté clérical que je ne lui connaissais pas auparavant et Magid, quant à lui, demande aux lecteurs de Watanide prononcer pour les «opprimés " la prière miraculeuse grâce à laquelle l'Église a affronté «tous les aventuriers mamelouks et sandjaks qui ont été portés au pouvoir en Égypte ». J'ai été étonné qu'il ait oublié d'ajouter, à la liste des envahisseurs, les Français, les Anglais et les Américains !

Après avoir parlé du "volcan de la prière ", il traite, dans le numéro suivant, des martyrs de la foi qui apparaissent aux coptes derrière les saints autels (dans les églises et monastères anciens) et qui leur disent: "Vous êtes les héritiers de la résistance spirituelle, et les moments glorieux de l'histoire copte suscitent l'espoir dans le coeur des jeunes, qui deviennent les témoins de ce qu'a accompli le sang des martyrs... »

31 À quoi avez-vous pensé, professeur Magid ? Que Dieu vous garde en sa miséricorde !

\section{INDEX}

Mots-clés : Coptes, Fawda (Farag) 